\title{
REVISÃo DE LITERATURA Associação entre uso de álcool em indivíduos com AIDS e adesão ao tratamento antirretroviral: uma revisão da literatura
}

Association between the usage of alcohol by HIV patients and the adherence to the antiretroviral drug treatment: a literature review

Samuel Robson Moreira Rego', Daianny Macedo de Sousa Rego ${ }^{1}$

\section{RESUMO}

Objetivo: $O$ objetivo deste trabalho foi fazer uma revisão sistemática da literatura, utilizando a base de dados MedLine, sobre o tema: uso/abuso/dependência de álcool como um fator de risco à redução da adesão, à redução na supressão da carga viral ou ao pior desfecho clínico em pacientes com AIDS em uso de highly active antiretroviral therapy (HAART). Método: Foi realizada uma pesquisa sistemática na base de dados MedLine utilizando como unitermos "HAART", "adherence" e "alcohol", na busca de artigos que versassem sobre a temática: avaliação ou associação de uso/abuso/dependência de álcool e adesão/supressão da carga viral/ desfecho clínico nos pacientes em uso de terapia antirretroviral. Resultados: A busca resultou em 65 artigos. Contudo, apenas 21 deles contemplaram os critérios de inclusão e foram selecionados. Foi encontrada associação positiva entre uso/abuso/dependência de álcool e

\section{Palavras-chave}

Álcool, adesão, HAART, AIDS.

\section{Keywords}

Alcohol, adherence, HAART, AIDS. baixa adesão/baixa supressão da carga viral/pior desfecho clínico em 18 (85,7\%) artigos. Conclusão: $O$ uso/abuso/dependência de álcool é um fator de risco para baixa adesão/baixa supressão da carga viral/pior desfecho clínico nos indivíduos em uso de HAART.

\begin{abstract}
Objective: The purpose of this work was to make a systematic review of the literature, using the MedLine data basis, about the alcohol usage/abuse/dependence as a risk factor to the adherence reduction, the viral load reduction or even a worse clinical outcome in HIV patients in the usage of highly active antiretroviral therapy (HAART). Method: It was made a systematic research in the MedLine data basis using the words: "HAART", "adherence" and "alcohol" with the purpose of finding articles about the themes: evaluations or association between the alcohol usage/abuse/dependence and adherence/suppression of the viral load/clinical outcome in HIV patients in the usage of antiretroviral therapy. Results: The search found 65 articles. However, only 21 of this achieved the pre-established criteria and have been selected. It was found a positive association among the usage/abuse/dependence of the alcohol and the low adherence/low viral load suppression/worse clinical outcome in $18(85,7 \%)$ of the articles. Conclusion: We could conclude that the alcohol usage/abuse/dependence is a risk factor to the low adherence/low viral load suppression/worse clinical outcome in HIV patients in the usage of HAART.
\end{abstract}

1 Universidade Estadual do Piauí (UESPI).

Endereço para correspondência: Samuel Robson Moreira Rego Rua Félix Pacheco, 2159, sala 501 - 64001-160 - Teresina, PI Telefones: (86) 3232-9406/3226-2726

E-mail: samuelregoteresina@yahoo.com.br 


\section{INTRODUÇÃO}

Estar infectado pelo HIV já não é mais sinônimo de letalidade. Desde que a doença foi descoberta, no início da década de 1980, muitos avanços foram dados em relação à maior compreensão da doença e desenvolvimento terapêutico. Atualmente, uma combinação de medicamentos conhecida como HAART (highly active antiretroviral therapy) é indiscutivelmente reconhecida por tornar mais lento o curso da doença e prolongar a vida ${ }^{1,2}$. Entretanto, um novo problema emergiu com o desenvolvimento e o uso dos antirretrovirais: a adesão aos esquemas terapêuticos. Esses são bastante complexos tanto pela grande quantidade de comprimidos como também pelo grande número de tomadas ao dia, além dos vários efeitos adversos que estão associados à medicação. Somado a tudo isso, tem-se o fato de uma adesão próxima a 100\% ser requerida para o sucesso do tratamento. Enquanto em algumas doenças crônicas, como diabetes e hipertensão arterial, 80\%-90\% de adesão sejam considerados satisfatórios, tendo geralmente excelente controle da doença, somente um terço dos pacientes com níveis de adesão ao HAART semelhante consegue alcançar carga viral indetectável3.

A taxa de adesão encontrada em vários países tem oscilado entre 50\% e 75\%, e uma das prováveis causas para essa grande variação talvez seja a grande heterogeneidade na forma de mensurar a variável adesão. No Brasil, foram encontradas taxas de 69\% e 56,9\% em São Paulo e 72,3\% no Distrito Federal. A não adesão aos novos medicamentos antirretrovirais pode ser considerada uma ameaça tanto à efetividade do tratamento como para a disseminação de vírus-resistência. Portanto, identificar os fatores que limitam a adesão é de grande relevância para que medidas que melhorem a adesão possam ser implementadas pela equipe de saúde ${ }^{4-6}$.

Estudos demonstram que são fatores de risco para uma baixa adesão: tempo de tratamento muito longo, complexidade do esquema terapêutico, efeitos colaterais intensos, suporte social fraco, depressão, uso problemático de álcool e uso de drogas ilícitas ${ }^{7-10}$.

O consumo de álcool está associado com pior prognóstico em pacientes com AIDS. O seu uso problemático pode reduzir a adesão do paciente ao HAART, além de propiciar comportamentos de risco como uso de outras drogas e de aumentar a frequência de depressão. Segundo estudos, a frequência de uso abusivo de álcool em pessoas convivendo com HIV é bastante elevada e variou de $8 \%$ a 32\%. Apesar desses números, a maioria dos médicos não investiga problemas decorrentes do uso de álcool em seu pacientes ${ }^{10-16}$.

O objetivo deste trabalho foi fazer uma revisão sistemática da literatura, utilizando a base de dados MedLine, sobre o tema: uso/abuso/dependência de álcool como um fator de risco à redução da adesão, redução na supressão da carga viral ou pior desfecho clínico em pacientes com AIDS em uso de HAART.

\section{MÉTODO}

Foi realizada uma pesquisa na base de dados MedLine utilizando como unitermos as palavras: HAART and Adherence and Alcohol. Foram selecionados apenas artigos originais, prospectivos ou de caso-controle, e metanálises escritos em língua inglesa e que versassem sobre a temática: avaliação ou associação de uso/abuso/dependência de álcool e adesão/supressão da carga viral/desfecho clínico nos pacientes em uso de terapia antiretroviral (TARV). Artigos de revisão não sistemática e artigos de atualização foram excluídos. A seleção dos artigos foi realizada após a avaliação cuidadosa dos títulos e dos abstracts disponíveis.

\section{RESULTADOS}

A busca no MedLine utilizando as palavras "HAART", "adherence" e "alcohol" resultou em 65 artigos. Após avaliação dos idiomas, dos títulos e dos abstracts, apenas 21 artigos contemplaram os critérios de inclusão e foram selecionados. Os demais 44 foram rejeitados por não tratarem especificamente do tema proposto ou apresentarem algum critério de exclusão.

Dos 21 artigos selecionados, um era do tipo metanálise e os demais eram estudos prospectivos ou de caso-controle. Foi encontrada associação significativa entre uso/abuso/dependência de álcool e baixa adesão/baixa supressão da carga viral/pior desfecho clínico em 18 (85,7\%) artigos. Contudo, dois desses encontraram associação significativa apenas em mulheres, mas não em homens. Apenas $3(14,3 \%)$ artigos não encontraram associação significativa (Tabela 1).

Em relação aos estudos que não encontraram associação significativa, todos apresentaram limitação no tocante ao tamanho da amostra, bastante reduzido. Ademais, o de Parsons, publicado em 2008, avaliou apenas indivíduos com uso problemático de álcool e excluiu os não usuários e pessoas com outros problemas psiquiátricos, não constituindo, dessa forma, uma amostra que correspondesse à população de indivíduos com AIDS em uso de TARV. Além disso, nesse mesmo trabalho não foi encontrada associação significativa entre consumo de álcool e supressão da carga viral, mas foi encontrada associação entre consumo de álcool e baixa adesão à TARV. Esse mesmo autor encontrou associação significativa em dois outros artigos publicados em 2007. Em um desses artigos, escrito juntamente com Rosof e Mustanski, Parsons avaliou os subfatores do padrão de uso de álcool e demonstrou que entre os não aderentes é a quantidade de drinks consumidos pelo indivíduo que prediz se haverá ou não falhas na adesão ${ }^{17-21}$.

Outro estudo que não encontrou associação significativa, o de Hinkin, apresentou uma prevalência de baixa adesão em 77\% dos indivíduos com abuso/dependência de álcool. 
Apesar de essa frequência ter sido maior que entre os sem abuso/dependência de álcool, essa diferença não foi significativa $\left[\left(x^{2}(1,144)=0,73, P=0,58\right)\right]$. Todavia, o autor não menciona o número de indivíduos com critérios para abuso/ dependência de álcool, mas relata que a baixa frequência desse diagnóstico em sua amostra pode ter provocado um viés. Além disso, esse estudo também avaliou a presença de déficit cognitivo/executivo em idosos. O autor especula que isso, também, pode ter contribuído para a ausência de diferença significativa na adesão entre os grupos com e sem problemas com álcool ${ }^{20}$.

Por outro lado, Tucker22 analisou dados do HIV Cost and Services Utilization Study, uma amostra representativa de norte-americanos infectados pelo HIV e em tratamento nos Estados Unidos no início de 1996, e concluiu que indivíduos que fazem uso de álcool tendem a aderir menos do que não usuários. Ele avaliou que não apenas os bebedores pesados, mas também os bebedores moderados, podem ter uma redução no índice de adesão à terapia antirretroviral. Nesse estudo, também esteve disponível para avaliação a carga viral de 757 indivíduos, sendo encontrada uma associação significativa entre os não aderentes e baixa supressão da carga viral $(P=0,009)^{22}$.

Tabela 1. Relação de artigos que investigaram a associação entre uso/abuso/dependência de álcool e adesão/supressão de carga viral/desfecho clínico nos pacientes em uso de terapia antirretroviral

\begin{tabular}{llll}
\hline Referências bibliográficas & Associação & Tipodo estudo & $\mathbf{n}$ \\
\hline Parsons et al. $(2008)^{17}$ & Não significativa & Caso-controle & 275 \\
Parsons et al. $(2007)^{18}$ & Significativa & Caso-controle & 272 \\
Parsons et al. $(2007)^{19}$ & Significativa & Prospectivo & 143 \\
Hinkin et al. $(2004)^{20}$ & Não significativa & Caso-controle & 148 \\
Martini et al. $(2004)^{21}$ & Não significativa & Caso-controle & 214 \\
Tucker et al. $(2003)^{22}$ & Significativa & Prospectivo & 1.910 \\
Braithwaite et al. $(2007)^{23}$ & Significativa & Prospectivo & 2.702 \\
Hendershot et al. $(2009)^{24}$ & Significativa & Metanálise & 25.000 \\
Beyene et al. $(2009)^{25}$ & Significativa & Caso-controle & 422 \\
Protopopescu et al. $(2009)^{26}$ & Significativa & Prospectivo & 1.010 \\
Bonolo et al. $(2008)^{27}$ & Significativa & Prospectivo & 295 \\
Hicks et al. $(2007)^{28}$ & Significativa & Caso-controle & 659 \\
Pence et al. $(2007)^{29}$ & Significativa & Caso-controle & 198 \\
Peretti-Watel et al. $(2006)^{30}$ & Significativa & Caso-controle & 4.963 \\
Samet et al. $(2004)^{31}$ & Significativa & Prospectivo & 267 \\
Palepu et al. $(2004)^{32}$ & Significativa & Prospectivo & 349 \\
Palepu et al. $(2003)^{33}$ & Significativa & Caso-controle & 234 \\
Arnsten et al. $(2002)^{34}$ & Significativa & Prospectivo & 85 \\
Golin et al. $(2002)^{35}$ & Significativa & Prospectivo & 140 \\
Applebaum et al. $(2009)^{36}$ & Significativa* & Prospectivo & 67 \\
\hline Apenas em mulheres. $(2007)^{37}$ & Significativa* & Prospectivo & 1.944 \\
\hline
\end{tabular}

Em relação ao desfecho clínico, Braithwaite et al. ${ }^{23}$ avaliaram uma coorte com mais de 2.500 pessoas convivendo com o vírus HIV e estimou que o uso pesado de álcool ( $\geq 5$ doses/ dia de consumo) pode reduzir a sobrevida desses indivíduos, em média, em mais de três anos se o consumo é semanal e em mais de seis anos quando o consumo é diário. Também foi estimada uma redução na sobrevida dos indivíduos com uso moderado ( $<5$ doses/dia de consumo), sendo, em média, maior que um ano em pessoas com consumo semanal e maior que três anos quando o consumo é diário ${ }^{23}$.

Corroborando esses dados, uma metanálise recente realizada por Hendershot et al. ${ }^{24}$ reuniu participantes captados em 40 estudos encontrados na literatura. Nesse trabalho foi encontrada uma associação significativa entre uso de álcool e não aderência ao uso da TARV. De acordo com essa metanálise, bebedores têm índice de adesão de, aproximadamente, 50\% quando comparados a indivíduos que não fazem uso de álcool ${ }^{24}$.

\section{DISCUSSÃO}

O uso combinado dos antirretrovirais possibilitou redução significativa da mortalidade em indivíduos com AIDS. Contudo, a necessidade de alta taxa de adesão (> 95\%) para que a terapia seja bem-sucedida tem sido um grande obstáculo aos indivíduos em uso do HAART.

Somando-se a esse fato, várias situações podem interferir no correto uso da medicação. Entre esses obstáculos, o uso de álcool, que é um comportamento modificável, temse mostrado como um forte preditor de falhas na adesão e piora no desfecho clínico ${ }^{25-35}$.

Entretanto, dois estudos mostraram que o fator gênero pode ser uma variável que interfere na associação entre uso problemático de álcool e baixa adesão; as mulheres estariam mais suscetíveis a não aderir quando apresentam problemas com álcool do que os homens. Contudo, o estudo de Applebaum et al..$^{36}$ trabalhou com uma amostra muito reduzida, de forma que os resultados não podem ser generalizados para outras populações, e no estudo de Lazo a amostra de indivíduos do gênero masculino foi retirada de uma coorte realizada em quatro cidades americanas, incluindo apenas indivíduos MSM (men who have sex with men). Dessa forma, conclusões sobre o gênero e adesão também não podem ser estendidas para a população geral de pessoas com AIDS 36,37.

Apesar de não estar claro o motivo pelo qual o uso problemático de álcool afeta a adesão, parece estar bastante evidente que essa variável seja um fator de risco para o déficit de adesão ao HAART. Nessa revisão, encontrou-se que 85\% dos artigos que investigaram essa associação mostraram ser significativa a relação entre consumo de álcool e dificuldade no uso correto das medicações antirretrovirais. Contudo, não se investigou a relação entre intervenções terapêuticas em usuários de álcool e o seu impacto na adesão e no desfecho 
clínico dos pacientes com AIDS. Estudos desse tipo são necessários para que se possam planejar ações de saúde pública que visem melhorar a qualidade da assistência aos pacientes convivendo com o vírus HIV e que fazem uso de HAART.

\section{CONCLUSÃO}

O consumo de álcool, até mesmo em situações que não caracterizam uso nocivo ou dependência, tem-se mostrado ser um preditor de falha na adesão ao HAART em indivíduos com AIDS. Além disso, ele também é um fator de risco para baixa supressão da carga viral e pior desfecho clínico nesses indivíduos.

\section{REFERÊNCIAS}

1. Gir E, Vaichulonis CG, Oliveira MD. Adesão à terapêutica antirretroviral por indivíduos com HIV/AIDS assistidos em uma instituição do interior paulista. Rev Latino-Am Enfermagem. 2005;13(5):634-41.

2. Colombrine MRC, Lopes MHBM, Figueiredo RM. Adesão à terapia antirretroviral para HIV/ AIDS. Rev Esc Enferm, USP. 2006;40(4):576-81.

3. Cheever L. Adesão à terapia anti-retroviral e assistência aos pacientes HIV+ usuários de drogas. 4a Conferência Anual Aids Brasil. Universidade John Hopkins hopkins-aids.edu/ educational/events/brasil; 2000.

4. Seidl EMF, Melchíades A, Farias V, Brito A. Pessoas vivendo com HIV/AIDS: variáveis associadas à adesão ao tratamento antirretroviral. Cad Saude Publica. 2007;23(10):2305-16.

5. Nemes MIB, Carvalho HB, Souza MFM. Antiretroviral therapy adherence in Brazil. AIDS. 2004;18(Suppl 3):515-20.

6. Pinheiro CAT, De Carvalho Leite JC, Drachler ML, Silveira VL. Factors associated with adherence to antiretroviral therapy in HIV/AIDS patients: a cross-sectional study in southern Brazil. Braz J Med Biol Res. 2002;35(10):1173-8.

7. Gifford AL, Bormann JE, Shively MJ, Wright BC, Richman DD, Bozzette SA. Predictors of self-reported adherence and plasma HIV concentrations in patients on multidrug antiretroviral regimens. JAIDS. 2000;23(5):386-95.

8. Haubrich RH, Little SJ, Currier JS, Forthal DN, Kemper CA, Beall GN, et al. The value of patient-reported adherence to antiretroviral therapy in predicting virologic and immunologic response. California Collaborative Treatment Group. AIDS. 1999;13(9):1099-107.

9. Gordillo V, Del-Amo J, Soriano V, Gozález-Lahoz J. Sociodemographic and psychological variables influencing adherence to antiretroviral therapy. AIDS. 1999;13(13):1763-9.

10. Monreal MT, Da Cunha Rv, Trinca LA. Compliance to antiretroviral medication as reported by AIDS patients assisted at the university hospital of the Federal University of Mato Grosso do Sul. Braz J Infect Dis. 2002;6(1):8-14.

11. Galvan FH, Bing EG, Fleishman JA, London AS, Caetano R, Burnam MA, et al. The prevalence of alcohol consumption and heavy drinking among people with HIV in the United States: results from HIV cost and services utilization study. I Stud Alcohol. 2002;63(2):179-86.

12. Lefevre F, O'Leary B, Moran M, Mossar M, Yarnold PR, Martin GJ, et al. Alcohol consumption among HIV-infected patients. J Gen Intern Med. 1995;10:458-60.

13. Kim TW, Palepu A, Cheng DM, Libman H, Saitz R, Samet JH. Factor associated with discontinuation of antiretroviral therapy in HIV-infected patients with alcohol problems. AIDS Care. 2007;19(8):1039-47.

14. Colibazzi T, Hsu TT, Gilmer WS. Human immunodeficiency virus and depression in primary care: a clinical review. Prim Care Companion J Clin Psychiatry. 2006:8(4):201-11.

15. Horton NJ, Meli S, Freedberg KA, Palepu A. Alcohol consumption and antiretroviral adherence among HIV-infected persons with alcohol problems. Alcohol Clin Exp Res. 2004;28(4):572-7.

16. Garcia R, Badaró R, Netto EM, Amorin FS, Ramos A, Vaida F, et al. Cross-sectional study to evaluate factors associated with adherence to antiretroviral therapy by Brazilian HIVinfected patients. AIDS Res Hum Retroviruses. 2006;22(12):1248-52.
17. Parsons JT, Rosof E, Mustanski B. Medication adherence mediates the relationship between adherence self-efficacy and biological assessments of HIV health among those with alcohol use disorders. AIDS Behav. 2008;12(1):95-103.

18. Parsons JT, RosofE, Mustanski B. Patient-related factors predicting HIV medication adherence among men and women with alcohol problems. J Health Psychol. 2007;12(2):357-70.

19. Parsons JT, Golub SA, Rosof E, Holder C. Motivational interviewing and cognitive-behavioral intervention to improve HIV medication adherence among hazardous drinkers: a randomized controlled trial. J Acquir Immune Defic Syndr. 2007;46(4):443-50.

20. Hinkin CH, Hardy DJ, Mason KI, Castellon SA, Durvasula RS, Lam MN, et al. Medication adherence in HIV-infected adults: effect of patient age, cognitive status, and substance abuse. AIDS. 2004;18(Suppl 1):S19-25.

21. Martini M, Recchia E, Nasta P, Castanotto D, Chiaffarino F, Parazzini F, et al. llicit drug use: can it predict adherence to antiretroviral therapy? Eur J Epidemiol. 2004;19(6):585-7.

22. Tucker JS, Burnam MA, Sherbourne CD, Kung FY, Gifford AL. Substance use and mental health correlates of nonadherence to antiretroviral medications in a sample of patients with human immunodeficiency virus infection. Am J Med. 2003;114(7):573-80.

23. Braithwaite RS, Conigliaro J, Roberts MS, Shechter S, Schaefer A, McGinnis K, et al. Estimating the impact of alcohol consumption on survival for HIV+ individuals. AIDS Care. 2007:19(4):459-66.

24. Hendershot CS, Stoner SA, Pantalone DW, Simoni JM. Alcohol use and antiretroviral adherence: review and meta-analysis. J Acquir Immune Defic Syndr. 2009;52(2):180-202.

25. Beyene KA, Gedif T, Gebre-Mariam T, Engidawork E. Highly active antiretroviral therapy adherence and its determinants in selected hospitals from south and central Ethiopia. Pharmacoepidemiol Drug Saf. 2009;18(11):1007-15.

26. Protopopescu C, Raffi F, Roux P, Reynes J, Dellamonica P, Spire B, et al. Factors associated with non-adherence to long-term highly active antiretroviral therapy: a 10 year follow-up analysis with correction for the bias induced by missing data. J Antimicrob Chemother. 2009;64(3):599-606

27. Bonolo Pde F, Machado CJ, César CC, Ceccato MG, Guimarães MD. Vulnerability and nonadherence to antiretroviral therapy among HIV patients, Minas Gerais State, Brazil. Cad Saude Publica. 2008;24(11):2603-13.

28. Hicks PL, Mulvey KP, Chander G, Fleishman JA, Josephs JS, Korthuis PT, et al. The impact of illicit drug use and substance abuse treatment on adherence to HAART. AIDS Care. 2007:19(9):1134-40.

29. Pence BW, Miller WC, Gaynes BN, Eron JJ Jr. Psychiatric illness and virologic response in patients initiating highly active antiretroviral therapy. J Acquir Immune Defic Syndr. 2007:44(2):159-66

30. Peretti-Watel P, Spire B, Lert F, Obadia Y. VESPA Group. Drug use patterns and adherence to treatment among HIV-positive patients: evidence from a large sample of French outpatients (ANRS-EN12-VESPA 2003). Drug Alcohol Depend. 2006;82(Suppl 1):S71-9.

31. Samet JH, Horton NJ, Meli S, Freedberg KA, Palepu A. Alcohol consumption and antiretroviral adherence among HIV-infected persons with alcohol problems. Alcohol Clin Exp Res. 2004;28(4):572-7.

32. Palepu A, Horton NJ, Tibbetts N, Meli S, Samet JH. Uptake and adherence to highly active antiretroviral therapy among HIV-infected people with alcohol and other substance use problems: the impact of substance abuse treatment. Addiction. 2004;99(3):361-8.

33. Palepu A, Tyndall MW, Li K, Yip B, O'Shaughnessy MV, Schechter MT, et al. Alcohol use and Incarceration adversely affect HIV-1 RNA suppression among injection drug users starting antiretroviral therapy. J Urban Health. $2003 ; 80(4): 667-75$.

34. Arnsten JH, Demas PA, Grant RW, Gourevitch MN, Farzadegan H, Howard AA, et al. Impact of active drug use on antiretroviral therapy adherence and viral suppression in HIV-infected drug users. J Gen Intern Med. 2002;17(5):377-81.

35. Golin CE, Liu H, Hays RD, Miller LG, Beck CK, Ickovics J, et al. A prospective study of predictors of adherence to combination antiretroviral medication. J Gen Intern Med. 2002;17(10):756-65.

36. Applebaum AJ, Richardson MA, Brady SM, Brief DJ, Keane TM. Gender and other psychosocial factors as predictors of adherence to highly active antiretroviral therapy (HAART) in adults with comorbid HIV/AIDS, psychiatric and substance-related disorder. AIDS Behav. 2009:13(1):60-5.

37. Lazo M, Gange SJ, Wilson TE, Anastos K, Ostrow DG, Witt MD, et al. Patterns and predictors of changes in adherence to highly active antiretroviral therapy: Iongitudinal study of men and women. Clin Infect Dis. 2007:45(10):1377-85. 